\title{
EMISSION METHODS OF EXPERIMENTAL INVESTIGATIONS OF ION VELOCITIES IN VACUUM ARC PLASMAS
}

\author{
A.S. Bugaev, V.I. Gushenets, A.G. Nikolaev, E.M. Oks, \\ G.Yu. Yushkov, A. Anders*, I.G. Brown* \\ High Current Electronics Institute Russian Academy of Sciences \\ 4 Academichesky ave., Tomsk \\ Russia 634055 \\ *Lawrence Berkeley National Laboratory, University of California, \\ 1 Cyclotron Road, Mailstop 53, Berkeley, California \\ USA, 94720
}

\begin{abstract}
This paper is devoted to an investigation of the directional velocities of the ions generated in cathode spots of vacuum arc discharges. Using emission methods of studying the processes in a vacuum arc discharge, which involve the determination of the parameters and characteristics of the discharge plasma by analyzing the ion current extracted from the plasma and the ion charge states, the velocities of ions have been determined for the majority of cathode materials available in the periodic table. Is has been shown that at a low pressure of the residual gas in the discharge gap the directional velocities of the ions do not depend on the ion charge state. Comparison of the data obtained with calculated values allows the conclusion that the acceleration of ions in a vacuum arc occurs by the magnetohydrodynamic mechanism.
\end{abstract}

\section{Introduction}

Among the key experimental facts established in studying the vacuum arc discharge $[1,2]$, an important one is the existence of directional flows of ions emitted by cathode spots and moving toward the anode with an energy exceeding the operating voltage of the discharge $[3,4]$. Now there is no consensus of opinion regarding the physical mechanism of the acceleration of ions, which is, first of all, due to the inconsistency of the available experimental data.

In spite of the fact that Davis and Miller [5] have found that the ion velocity increases in direct proportion with its charge, in some other experiments, for example, in the experiment of Tsuruta et al. [6], under certain conditions the measured velocities of ions practically did not depend on the ion charge state.

The emission methods developed by us to investigate the processes in a vacuum arc discharge $[7,8]$ involve the determination of plasma parameters and characteristics 
based on the analysis of the ion current extracted from the plasma and its charge states. In this paper, the results of the determination of directional velocities of ions in a vacuum arc and their comparison with predicted values are given, allowing a conclusion about the mechanism of the acceleration of ions in a vacuum arc.

\section{The emission method for investigating ion velocities}

The basic methodical difficulties arising in studying the parameters of the plasma of a vacuum arc are related to the fact that the cathode spot occupies a random position and moves with a velocity of $\sim 10^{4} \mathrm{~cm} / \mathrm{s}$ over the cathode surface, it is small in size $\left(<10^{-1}\right.$ $\mathrm{cm}$ ), and the plasma density in the region adjacent to the cathode spot is over $10^{18} \mathrm{~cm}^{3}$. These features of the cathode spot as a physical object impede its experimental investigation. In this connection, methods of corpuscular diagnostics, in particular the emission method [7,8], seem to offer, perhaps, a solely probable approach for studying experimentally the processes in a cathode spot. The idea of the method is to study of the response of the charge state distribution of the ions extracted from the emission boundary located far away from the cathode spots of a vacuum arc to the action of which the discharge is subjected. Such an action may be the abrupt change in vacuum arc current resulting in the death of existing cathode spots or birth of new ones.

The electrode arrangement of the experimental setup is given in Fig. 1. The vacuum arc discharge was initiated in a discharge gap between cathode 1 and anode 3 . The duration and current of the discharge pulse were 200-500 $\mu$ s and 100-500 s, respectively. During the operation of the arc, cathode material plasma 4 emitted by cathode spots filled the cavity of the anode. There were emission holes in the face surface of the anode designed for extraction of ions from the plasma. The extracted ions were accelerated in multiaperture acceleration/deceleration system 5 by dc voltage of $10-30 \mathrm{kV}$. Subsequently the charge state constitution of ion flow 6 was analyzed by a time-of-flight mass spectrometer $[9,10]$.

In over $150 \mu$ s from the initiation of the vacuum arc, when all its basic parameters can be considered steady-stated, an additional current pulse was applied to the discharge gap or the power supply of the discharge was closed by a fast switch [11]. In the first

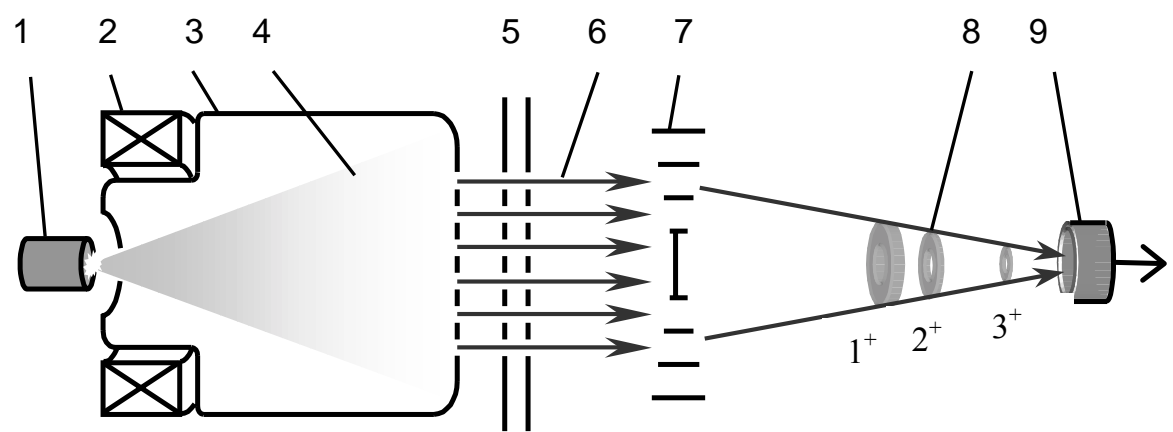

Figure 1. The electrode arrangement of the setup for measuring ion velocities: 1 - cathode; 2 - solenoid; 3 - anode; 4 - vacuum arc plasma flow; 5 - acceleration electrodes; 6 - ion beam; 7 - spectrometer gate; 8 ions of various charge state; 9 - Faraday cup. 


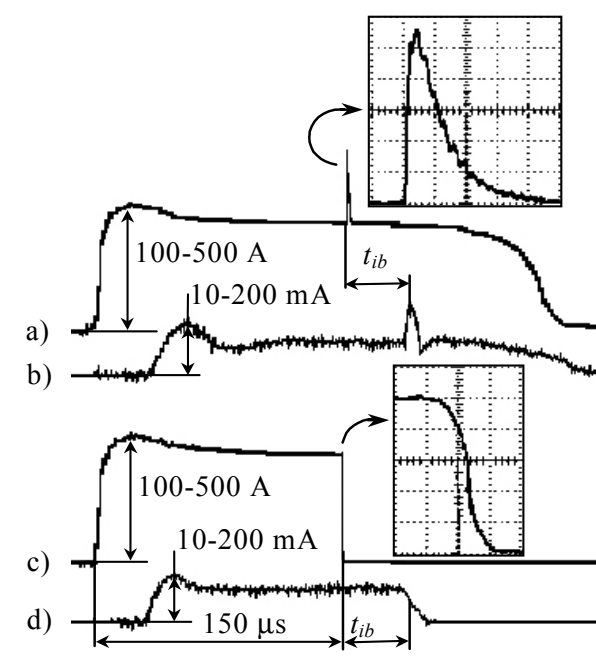

Figure 2. Oscillograms of the vacuum arc $(a, c)$ and ion beam currents $(b, d)$ in cases of a burst $(a, b)$ and cutoff $(c, d)$ of a current of an arc. Oscillograms of the arc current recorded on application of a current burst and during current cutoff are given in the insets: $1 \mu \mathrm{s} / \mathrm{div}, 25 \mathrm{~A} / \mathrm{div}$.

case, the arc current increased by 50-150 A within 2-4 $\mu$ s, while in the second one it decreased to zero in about $2 \mu \mathrm{s}$, leading to extinction of the discharge (Fig. 2). In what follows, when describing the mentioned changes in arc current, the term's "current burst" and "current cutoff" will be used.

Attention is drawn to the fact that both in the case of an arc current burst and in the case of its cutoff the response of the ion beam current to the mentioned perturbations of the arc current was observed after some time interval $t_{\mathrm{ib}}$. The measured values of $t_{\mathrm{ib}}$ depend on the cathode material being $8 \mu$ s for the lightest material $(\mathrm{C})$ and $40 \mu$ s for the heaviest one (Bi). At the same time, the travel times for the ions of these elements in the acceleration gap and in the drift space were less than 1 and $3 \mu \mathrm{s}$, respectively. It is obvious that this considerable difference in $t_{\mathrm{ib}}$ is due to the motion of the ions in the plasma from the cathode region, in which they have been generated, to the emission grid. By the change in current of each ion species it was possible to determine the time after the action on the vacuum arc in which the change in the emission current extracted from the discharge plasma occurred and thus find the velocity of motion of ions from the vacuum arc cathode spot to the emission surface.

\section{Influence of the charge of ions on their directional velocity in plasma}

Typical dependencies of the current on the time after the application of an additional current burst to the discharge gap for ions of different charge state obtained for the case of a vacuum arc with a magnesium cathode are given in Fig. 3. The same figure presents similar dependencies for the case the arc current was cut off. It should be noted that when a current burst is applied to the discharge gap, the currents of ions of each charge state reach a maximum at the same time, while in the case the discharge current is cut off, the fall in current occurs identically for variously charged ions. These features were observed for all cathode materials used in the experiment, namely, $\mathrm{Mg}, \mathrm{Al}, \mathrm{Ti}, \mathrm{Zr}$, $\mathrm{Cu}, \mathrm{Pb}$, and $\mathrm{Bi}$.

Both in the case of an arc current burst and in the case of its cutoff the response of the ion current shows up in a certain time $t$. Since the basic processes of ionization of the cathode material in a vacuum arc occur near cathode spots, at distances which are not over $1 \mathrm{~mm}$ from the cathode surface and much smaller than the cathode-anode 
separation $L_{\mathrm{c}-\mathrm{a}}$, and are followed only by adiabatic expansion of the plasma flare [12], the velocity of ions in the plasma, $v_{i}$, can be determined by the formula:

$$
v_{\mathrm{i}}=\frac{L_{\mathrm{c}-\mathrm{a}}}{t-\left(L_{3}+2 d_{\mathrm{accel}}\right) \cdot \sqrt{M_{\mathrm{i}} / 2 Q e U_{\text {accel }}}},
$$

where $V_{\text {accel }}$ is the amplitude of the accelerating voltage; $M_{i}$ and $e Q$ are the mass and charge of the ions; $d_{\text {accel }}$ is the width of the acceleration gap; $L_{3}$ is the distance from the acceleration electrode to the gate of the mass spectrometer. The value of $t$ in the case of an arc current burst was determined as the time between the maxima of the discharge and the ion currents, while in the case of current cutoff the value of $t$ was taken as the interval between the maxima of the time dependencies of these currents differentiated with respect to time. The investigations performed have shown that the velocities of directional motion of ions of the same material, but of different charge are practically identical. Differentiating the time dependencies of ion current obtained after the closure of the discharge gap, we can obtain the function of distribution of the ions by directional velocities. Thus we obtain that not only the maxima of the velocity distributions for different ionic species practically coincide with each other, but the distributions themselves as well. A magnetic field created in the discharge gap increased the directional velocities of ions; however, the currents of ions of different charge for each cathode material varied proportionally.

The data obtained do not agree, first of all, with the experimental results of Tsuruta et al. [5] who measured the dependence of the velocity of ions, $v_{i}$, on their charge. It is obvious that this disagreement should be due to different experimental conditions. Essential differences were that in the experiment [5] the arc discharge operated during $t=0.5 \mathrm{~s}$, while in the experiment under consideration $t$ was shorter than $0.5 \mathrm{~ms}$ and that in [5] the discharge device was evacuated with an ion pump at a rate of only $20 \mathrm{l} / \mathrm{s}$. The long duration of the discharge pulse and the rather small evacuation rate provided by the pump used in combination with the "falling" dependence of the evacuation rate quantity on pressure inherent in this type of pump could have the result that the pressure in the discharge system during the operation the vacuum arc was much higher than that reported in [5].
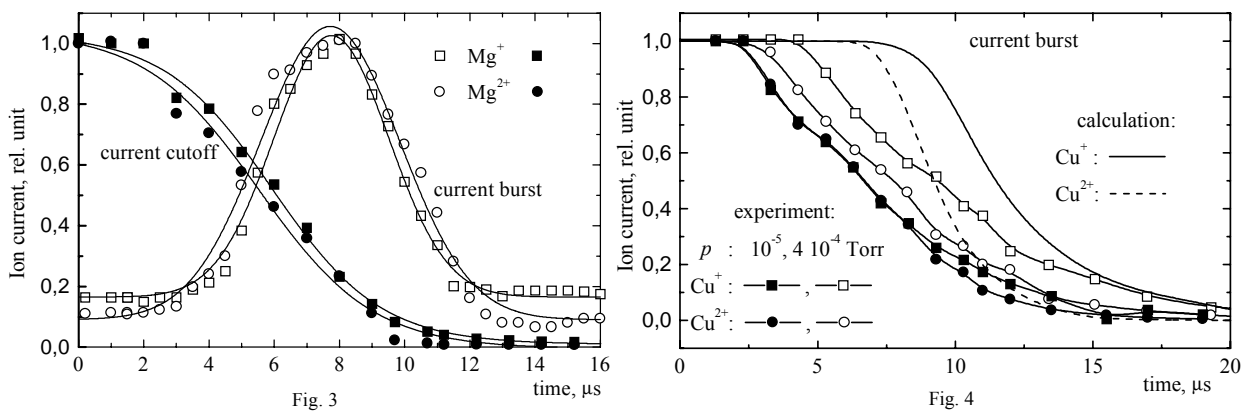

Figure 3. Typical dependencies of the current on the time after the application of an additional current burst to the discharge gap for ions of different charge state. Cathode $-\mathrm{Mg}$.

Figure 4. Dependencies of the ion current on the time after the cutoff of the arc current for $\mathrm{Cu}^{+}$and $\mathrm{Cu}_{2}^{+}$ ions. 
To verify this hypothesis, measurements of the velocities of ions were carried out with forced supply of gas in the discharge gap. An increase in pressure $p$ of the gas as it was supply into the discharge gap resulted in an increase in the time at which the response of the ion current to a perturbation of the arc. At the same time, the effect of an increase in $p$ was different for different charge states. As an example, we present the dependencies of the ion current on the time after the cutoff of the arc current for $\mathrm{Cu}^{+}$ and $\mathrm{Cu}_{2}{ }^{+}$ions (Fig. 4) for the case where Ar was supplied into the discharge system. In the same figure, the dependencies calculated based on the data obtained by the authors of [5] are given. It can be seen that the tendency in changing the dependencies with increasing gas pressure is toward a disproportionate change in the velocity of singly and doubly charged ions and as the pressure is increased, the dependencies approach those that should be observed according to the data of [5]. Unfortunately, in the test discharge system, owing to the necessity of maintenance of the electrical strength of the acceleration gap, the peak pressure was limited. However, the disproportionate change in velocities of variously charged ions has been observed unambiguously for various cathode materials and various gases supplied into the discharge gap.

\section{Directional velocities of ions for various cathode materials}

Since, as has been well established, the directional velocities of various ionic species are practically identical for $p<5 \cdot 10^{-5}$ Torr, the use of a time-of-flight spectrometer, intended for separation of the ionic component of a plasma by species, was not necessary for further investigation of directional velocities of ions. Detailed investigations of the dependence of the directional velocities of ions on the arc operating voltage were carried out with the use of the discharge system of the Mevva-5 ion source [13]. These experiments featured a slower modulation of the arc current pulse with characteristic oscillation time $t \gg 50 \mu$ s and amplitude not exceeding $30 \%$ of the pulse current and the use of a flat single Langmuir probe for measuring the ionic component of the plasma. The velocity of ions in the given experiments was estimated as $v_{i}=L_{\mathrm{c}-\mathrm{p}} / \Delta t$, where $L_{\mathrm{c}-\mathrm{p}}$ is the distance from the cathode to the probe surface and $\Delta t$ is the time shift between the oscillations of the discharge and probe currents (Fig. 5). The measured velocities of ions for various cathode materials, including the majority of


conductive materials of the periodic table, and the arc operating voltages are given in Table 1.

Figure 5. Oscillograms of the operating voltage $(1-20 \mathrm{~V} / \mathrm{div}$.), the ion current onto the probe $(2-0.1 \mathrm{~A} / \mathrm{div}$.$) and the discharge current$ $(3-200 \mathrm{~A} /$ div. $)$ for a vacuum arc with a tantalum cathode. Distance from the cathode to the probe: $24 \mathrm{~cm}$. The shift between the oscillations of the arc current and the ion current onto the probe was $20 \mu \mathrm{s}$, which corresponds to $v_{i}$ of tantalum ions, $1.2 \cdot 10^{6} \mathrm{~cm} / \mathrm{s}$. 
Table 1. Measurements of directional ion velocities. $V_{\mathrm{d}}$ is the arc operation voltage, $<Q>$ is the average ion charge, $T_{e}$ is the plasma electron temperature [14], $I_{\mathrm{d}}=250 \mathrm{~A}, p<7 \cdot 10^{-7}$ Torr.

\begin{tabular}{|c|c|c|c|c|c|c|c|c|c|}
\hline $\begin{array}{c}\text { Materi } \\
\text { al }\end{array}$ & $\begin{array}{c}\mathrm{V}_{\mathrm{i}} \cdot 10^{-6}, \\
\mathrm{~cm} / \mathrm{s}\end{array}$ & $\mathrm{V}_{\mathrm{d}}, \mathrm{V}$ & $<\mathrm{Q}>$ & $\mathrm{T}_{\mathrm{e}}, \mathrm{eV}$ & \begin{tabular}{|c|} 
Materi \\
al \\
\end{tabular} & $\begin{array}{c}\mathrm{v}_{\mathrm{i}} \cdot 10^{-6} \\
\mathrm{~cm} / \mathrm{s}\end{array}$ & $\mathrm{V}_{\mathrm{d}}, \mathrm{V}$ & $<\mathrm{Q}>$ & $\mathrm{T}_{\mathrm{e}}, \mathrm{eV}$ \\
\hline $\mathrm{Li}$ & 2.3 & 23.5 & 1 & 2 & $\mathrm{Cd}$ & 0.7 & 14.7 & 1.3 & 2.1 \\
\hline $\mathrm{C}$ & 1.7 & 31 & 1 & 2 & In & 0.6 & 16 & 1.3 & 2.1 \\
\hline $\mathrm{Mg}$ & 2 & 18.6 & 1.5 & 2.1 & $\mathrm{Sn}$ & 0.7 & 17.4 & 1.5 & 2.1 \\
\hline $\mathrm{Al}$ & 1.5 & 22.6 & 1.7 & 3.1 & $\mathrm{Ba}$ & 0.8 & 16.5 & 2 & 2.3 \\
\hline $\mathrm{Si}$ & 1.5 & 21 & 1.4 & 2 & $\mathrm{La}$ & 0.7 & 18.7 & 2.2 & 1.4 \\
\hline $\mathrm{Ca}$ & 1.4 & 20.5 & 1.9 & 2.2 & $\mathrm{Ce}$ & 0.8 & 17.6 & 2.1 & 1.7 \\
\hline $\mathrm{Sc}$ & 1.5 & 21.6 & 1,8 & 2.4 & $\operatorname{Pr}$ & 0.8 & 20.5 & 2.3 & 2.5 \\
\hline $\mathrm{Ti}$ & 1.5 & 22.1 & 2 & 3.2 & $\mathrm{Nd}$ & 0.8 & 19.2 & 2.2 & 1.6 \\
\hline V & 1.6 & 22.7 & 2,1 & 3.4 & $\mathrm{Sm}$ & 0.8 & 18.8 & 2.1 & 2.2 \\
\hline $\mathrm{Cr}$ & 1.6 & 22.7 & 2,1 & 3.4 & $\mathrm{Gd}$ & 0.8 & 20.4 & 2.2 & 1.7 \\
\hline $\mathrm{Fe}$ & 1.3 & 21.7 & 1.8 & 3.4 & $\mathrm{~Tb}$ & 0.8 & 19.6 & 2.2 & 2.1 \\
\hline $\mathrm{Co}$ & 1.2 & 21.8 & 1.7 & 3 & Dy & 0.8 & 19.8 & 2.3 & 2.4 \\
\hline $\mathrm{Ni}$ & 1.2 & 21.7 & 1,8 & 3 & Ho & 0.9 & 20 & 2.3 & 2.4 \\
\hline $\mathrm{Cu}$ & 1.3 & 22.7 & 2 & 3.5 & $\mathrm{Er}$ & 0.9 & 19.2 & 2.3 & 2 \\
\hline $\mathrm{Zn}$ & 1 & 17.1 & 1.2 & 2 & $\mathrm{Hf}$ & 1 & 23.3 & 2.9 & 3.6 \\
\hline $\mathrm{Ge}$ & 1.1 & 20 & 1.4 & 2 & $\mathrm{Ta}$ & 1.2 & 28.6 & 2.9 & 3.7 \\
\hline $\mathrm{Sr}$ & 1.2 & 18.5 & 2 & 2.5 & W & 1.1 & 28.7 & 3.1 & 4.3 \\
\hline Y & 1.3 & 19.9 & 2,3 & 2.4 & Ir & 1.1 & 25.5 & 2.7 & 4.2 \\
\hline $\mathrm{Zr}$ & 1.5 & 22.7 & 2.6 & 3.7 & $\mathrm{Pt}$ & 0.8 & 23.7 & 2.1 & 4 \\
\hline $\mathrm{Nb}$ & 1.6 & 27.9 & 3 & 4 & $\mathrm{Au}$ & 0.7 & 19.7 & 2 & 4 \\
\hline Mo & 1.7 & 29.5 & 3.1 & 4.5 & $\mathrm{~Pb}$ & 0.6 & 17.3 & 1.6 & 2 \\
\hline $\mathrm{Rh}$ & 1.5 & 23.8 & 1.8 & 4.5 & $\mathrm{Bi}$ & 0.5 & 14.4 & 1.2 & 1.8 \\
\hline $\mathrm{Pd}$ & 1.2 & 23.5 & 1.9 & 3.5 & Th & 1.0 & 23.3 & 2.9 & 2.4 \\
\hline $\mathrm{Ag}$ & 1.1 & 22.8 & 2.1 & 4 & $\mathrm{U}$ & 1.1 & 23.5 & 2.3 & 3.4 \\
\hline
\end{tabular}

\section{Analysis of experimental results}

There is reason to believe that the directional velocities of ions of lower charge decrease with increasing pressure more substantially due to collisions of the ions with the neutrals of the fill gas. At these collisions, owing to the nonresonance charge exchange of the ions of the cathode material with the gas neutrals, there occurs a decrease in ion charge. Thus, the singly charged ions are ions that have experienced more collisions with neutrals and consequently have a lower kinetic energy and a charge. Indirect evidence in support of this supposition is the experimental fact that the total ion current decreases as the pressure is increased due to the supply of gas into the discharge gap and that the effect of deceleration of ions is more pronounced if the fill gas has a greater specific mass.

The magnetohydrodynamic two-fluid model of the cathode flare plasma consisting of electrons and ions of medium charge number $<\mathrm{Q}>$, which expands adiabatically after explosive formation of an "ecton" has been developed by G. A. Mesyats and co-workers 
[15-17]. According to the results of their investigations [17], at a great distance from the cathode spot, the velocity of expansion of a cathode flare equal to the directional velocity of ions, $v_{i}$, can be determined as

$$
v_{\mathrm{i}}=(2 /(\gamma-1)) \cdot \sqrt{\gamma\left(<Q>T_{\mathrm{e}}+T_{\mathrm{i}}\right) / M_{\mathrm{i}}},
$$

where $\gamma$ is the adiabatic exponent equal to 5/3. Putting, as in [17], $T_{e} \approx T_{i}$ and substituting into expression (2) the values of $\left\langle Q>\right.$ and $T_{e}$ from Table 1 , we can estimate the directional velocities of ions. The resulting estimates are compared with experimental data in Fig. 6. The rather high degree of agreement between the calculations and measurements allows the conclusion that the observed ion velocities are achieved mainly due to the magnetohydrodynamic mechanism of acceleration, and expression (2) explains the experimental dependence of $v_{i}$ on $<Q>$ and $M_{i}$ as well as (since $T_{e} \propto V_{\mathrm{d}}$ ) on $V_{\mathrm{d}}$. The magnetohydrodynamic model of the acceleration of ions in the cathode region of a vacuum arc [8], specially proposed to interpret the results of the present investigations, is based on the idea that the acceleration of the cathode material goes on after its transition to the state of a completely ionized collisional plasma. As in [15-17], it is assumed that the reason for the acceleration of ions is the expansion of the plasma into vacuum under conditions of constant inflow of energy due to Joule heating. By comparison of the model predictions with experimental data it has been shown that the acceleration of the cathode material occurs in the main when the material is in the state of ideal completely ionized plasma, through its expansion into vacuum, and the velocities of ions at a considerable distance from the cathode spot of the vacuum arc given by

$$
v_{\mathrm{i}}=3,5 \cdot \sqrt{\gamma<Q>T_{\mathrm{e}} / M_{\mathrm{i}}}
$$

The predicted values of velocities are also given in Fig. 6. Good correlation with measurements and with the calculations using expression (2) is observed. An important feature of these investigations is the opportunity to calculate directional velocities for ions of various charge states. Based on the calculations it has been established that the velocity of ions of various charge states can differ from their common magnetohydrodynamic velocity only by a few percent, which is confirmed by the

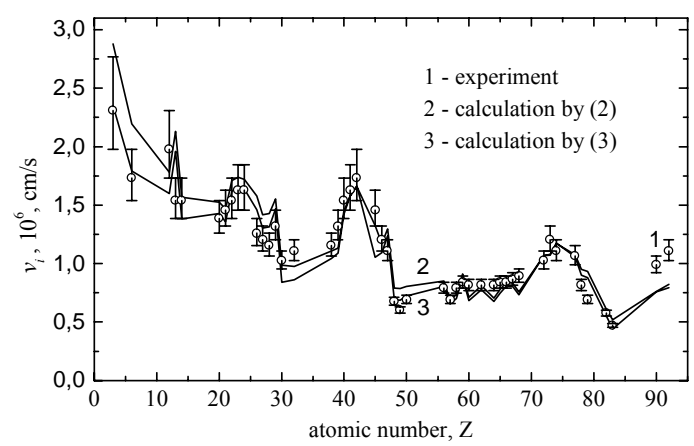

Figure 6. The predicted values of velocities. obtained experimental data.

In conclusion it is should be noted that, according to the above magnetohydrodynamic models, the factors that determine the directional velocity of ions should be the mass of the ions, their mean charge, and the electron temperature, and this is in complete agreement with the obtained experimental results.

The

investigations of directional

velocities performed allow us to obtain an unambiguous relation between the density of 
the saturation ion current onto a flat Langmuir probe located perpendicularly to the plasma flow and the parameters of the plasma:

$$
j_{i}=e<Q>n v_{i}=e<Q>n\left(20 T_{e}<Q>/ M_{i}\right)^{1 / 2} .
$$

The use of expression (4) is more justified than the frequently used (see, e.g., [13], page 344) estimation of the plasma parameters based on the well-known Bohm formula [18] derived on the assumption that the ions of a vacuum arc move with the sound velocity. At the same time, from the experimental results obtained it follows that the directional velocities of ions exceed substantially the ion sound velocities. It can easily be shown that the use of Bohm's formula for the definition of the plasma density by the ion current onto a flat probe gives a value underestimated more than three times.

\section{Conclusions}

The investigation results presented in this paper can be summarized as follows:

1. With the use of the emission method for measuring the directional velocities of ions of the vacuum arc plasma it has been shown that these velocities have values of the order of $10^{6} \mathrm{~cm} / \mathrm{s}$, depending on the cathode material and practically identical for differently charged ions. The working gas supplied into the discharge gap reduces the directional velocities of ions, and this decreased is more pronounced for lower charges.

2. Based on the results of investigations carried out for a variety of cathode materials, it has been shown that the value of the steady-stated operating voltage of a vacuum arc discharge determines the directional velocities of ions. The established experimental facts in combination testify to the development of the processes of acceleration of ions in a small near-cathode region where an intense energy exchange occurs both between the electronic and ionic subsystems of the plasma and between variously charged ions.

3. Based on the experimental and predicted values of the directional velocities of ions, comparing the measured and predicted dependencies of the velocities the mass of ions for various materials and the mean charge of the ions and the electron temperature, one can state that the magnetohydrodynamic mechanism is dominant in the acceleration of ions, and the reason for the acceleration is the Joule heating of the plasma and the energy transfer from its electronic subsystem to the ionic one.

4. Comprehensive measurements of the directional velocities of the ions of the vacuum arc plasma have been performed for the majority of metals of the periodic table. The experimental data obtained are of great importance not only for understanding the physical nature of the given type of discharge, but also in the practical sense and can be used both in the development of various devices based on vacuum arcs and for the optimization of technological processes which utilize the plasma of the given type of discharge.

\section{Acknowledgments}


The author extends his sincerest appreciation to E. M. Oks, A. S. Bugaev, V. A. Gushenets, and A. G. Nikolaev (Laboratory of Plasma Sources of the Institute of High Current Electronics, RAS) as well as to I. Brown and A. Anders (Lawrence Livermore National Lab., Berkeley, USA) and to I. A. Krinberg (Irkutsk State University) for their fruitful and constructive discussions of the investigation results and help in the performance of experiments.

The work has been supported in part by research contracts with Lawrence Livermore National Laboratory, Berkeley, USA, under the IPP Trust-1 AmericanRussian scientific cooperation program and by the Russian Foundation for Fundamental Research grant No. 99-02-18163.

\section{References:}

1. J.M. Lafferty (ed.), Vacuum arcs - theory and application. - Wiley, New York, 1980

2. G.A. Mesyats Ectons in a vacuum discharge: breakdown, the spark, and the arc.- Moscow, Nauka, 2000 .

3. A.A. Plutto,V.N. Ryzhkov, and A.T. Kapin, Zh. Eksp. Teor. Fiz. 47, 494 (1964).

4. E. Kobel, Phys. Rev. 36, 1636 (1930).

5. W.D. Davis, H.C. Miller, J. Appl. Phys. 40, 2212 (1969).

6. K. Tsuruta, K. Sekiya, and G. Watanabe, IEEE Trans. Plasma Sci. 25, 603 (1997).

7. A.S. Bugaev, V.I. Gushenets, A.G. Nikolaev, E.M. Oks, and G.Yu. Yushkov, Technical Physics. 45, 1135 (2000).

8. G.Yu. Yushkov, A.S. Bugaev, I.A. Krinberg, and E.M. Oks, Doklady Physics. 46, 307 (2001)

9. I.G. Brown, J.E. Galvin, R.A. MacGill, and R.T. Wright, Rev. Sci. Instrum. 58, 1589 (1987).

10. A.S. Bugaev, V.I. Gushenets, A.G. Nikolaev, E.M. Oks, and G.Yu. Yushkov, Izv. Vyssh. Ucheb. Zaved., Fizika, 2, 21 (2000)

11. G. Yushkov, Proc. $19^{\text {th }}$ Intern. Symp. on Discharges and Electrical Insulation in Vacuum. Xi'an, China, 2000, V. I, p. 260.

12. G.A. Mesyats Ectons - Ekaterinburg, Nauka, 1993

13. I.G. Brown (Ed.) The physics and technology of ion sources. - Wiley, New York, 1989.

14. A. Anders, Phys. Rev. E 55, 969 (1997).

15. E.A. Litvinov, In: High-power nanosecond pulsed sources of accelerated electrons. Ed. by G.A. Mesyats. - Novosibirsk, Nayka, 1974.

16. E.A. Litvinov, G.A. Mesyats, and D.I. Proskurovsky, Sov. Phys. Usp. 15, 102 (1975).

17. G.A. Mesyats, and D.I. Proskurovsky, Pulsed electrical discharge in vacuum. - Springer-Verlag, Berlin, 1989. 